\title{
THE PRODUCTION OF LIQUID AIR ON A LABORATORY SCALE
}

\author{
By J. W. Cook
}

\section{ABSTRACT}

The essentials of a plant producing liquid air by the Hampson process are the compressor, purifying train, and liquefier. The compressor, usually of four stages, delivers air at room temperature and approximately 3000 pounds per square inch. The compressed air purifying train consists of, first, a trap for receiving oil and water, and second, suitable containers charged with chemical reagents, such as sodium hydroxide, calcium chloride, or lime, for removing carbon dioxide and water vapor. The air thus compressed and purified is delivered to the liquefier, in which, after passing through a coil of copper tubing, the air is allowed to expand freely to approximately atmospheric pressure. Where this drop in pressure takes place there is a corresponding drop in the temperature of the air. The expanded air before leaving the liquefier is caused to circulate around the copper coil which contains the compressed air, thus cooling the coil and in turn the compressed air, so that on continuous operation a cycle of progressive cooling is maintained until the temperature ultimately reaches the liquefying point. The liquefier is so constructed that the air which is condensed to liquid is delivered into a receiving vessel. The gaseous air exhausted from the liquefier is returned to the intake of the compressor for succeeding cycles because it has been purified and when used repeatedly will be less exhausting on the purifying reagents.

\section{CONTENTS}

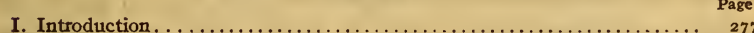

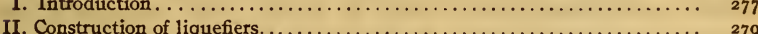

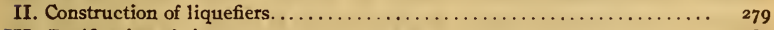

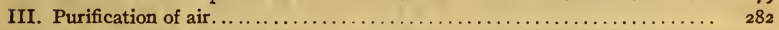

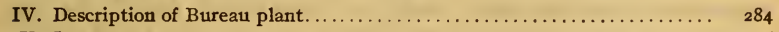

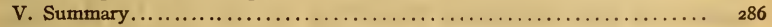

\section{INTRODUCTION}

The following is a brief account of the liquefaction of air on a laboratory scale by the Hampson process and a description of the apparatus now used at the Bureau of Standards. It is not the purpose of this paper to enter into the theory of the Hampson liquefier, which has been treated elsewhere, nor to furnish a working drawing whereby the apparatus may be duplicated, because details and methods of mechanical construction are rarely duplicated exactly by different constructors. It is the purpose to show the general methods of construction which will serve as a guide for others working along similar lines. 
It is well to call attention to the fact that the total cold available is determined by the initial temperature and pressure of the air at the point where it enters the regenerator and the exhaust pressure, which in practice is one atmosphere. It is independent of the internal construction or arrangement of the liquefier, of the material used in its construction, of the distribution of temperature in the regenerator coil, and of all other circumstances whatever, provided that the kinetic energies of the feed and the exhaust are negligible. This cold is used in the following three ways: (a) To offset the heat that leaks into the liquefier from outside, either through the insulation or along the metallic component parts of the liquefier; $(b)$ to cool the whole quantity of gas from its initial temperature to the slightly lower exhaust tenperature; $(c)$ to cool a fraction of the air from this exhaust temperature to the normal boiling point and then condense it into liquid. ${ }^{1}$

From these facts it is evident that to obtain the maximum amount of liquid air with a given expenditure of power a Hampson liquefier must be so constructed that the factors $(a)$ and $(b)$ are kept as small as possible. This results in a compromise, however, for by increasing the size of the regenerator indefinitely, which would make $(b)$ negligible by exhausting at practically the initial temperature, the size of the liquefier, over all, is necessarily increased, thereby increasing $(a)$ the heat leak. It is therefore apparent that, to be efficient, a liquefier should be as small, over all, as will exhaust air at a temperature just slightly below the initial temperature, which may be about room temperature or may be considerably lower if a precooler is used. The size of the regenerator coil required would be determined chiefly by the capacity of the compressor furnishing the air and by the temperature of the air entering the regenerator. Obviously, if a precooler us used, the size of the regenerator may be decreased.

In the foregoing discussion it has been assumed that the liquefier has been in operation long enough for complete thermal equilibrium to have been reached. Under these conditions the actual heat capacity would not be a factor. If a liquefier is to be run only intermittently for the production of small amounts of liquid at a time, the heat capacity of the liquefier will be an appreciable factor, influencing the length of time required to cool down the liquefier when starting, and effectiveness of heat interchange can be advantageously sacrificed in order to reduce the heat capacity.

1 Buckingham, B. S. Bull. 6, p. 125; 1909 (Sci. Paper No. 123). 


\section{CONSTRUCTION OF LIQUEFIERS}

In the liquefiers constructed at the Bureau the regenerator coil is a copper tube $\left(a a^{\prime}\right.$, Fig. $\left.\mathrm{r}\right)$ wound, starting at the top of the liquefier, in a spiral from outside to center for the first layer, then from center to the outside for the next layer, and so on down the liquefier, without multiplicity of tubes and without retracing in the reverse direction from bottom to top. ${ }^{2}$ Thus, all the incoming air must pass through the entire length of the regenerator. Fig. 2 is a photograph of a section of a regenerator in the course of construction. The spiral is wound on the brass forms shown in Fig. 2; the core is then slipped out of one of the forms to permit removing the two halves of this form from the coil. The form is then replaced on the core from the other end and the winding continued, the process being repeated until the entire length of a tube, usually about 25 feet, has been wound. The several coils are then pressed down into a series of nearly flat spirals, and as many sections as are required for the regenerator are joined with silver solder. It is more convenient to make the joints at the outermost turn of the spiral. The complete regenerator coil is then mounted permanently on a German-silver tube just strong enough to serve as a core and support the apparatus.

In winding the copper tubing it sometimes has a tendency to collapse or flatten. To prevent this it is convenient to fill a length of the tube with water and, with a hydraulic pump, maintain during the winding a constant hydrostatic pressure on the tube equal to the pressure at which the liquefier will be operated with air, usually 3000 pounds per square inch. If this process does not suffice, the tube may be filled with water, then frozen in a bath of brine and ice, and wound while the water in the tube is frozen. ${ }^{3}$ Annealed copper tubing will not burst on having water frozen once within it, and if winding is done before the ice has had time to melt a very true coil of small diameter may be made. Either of the above methods has the advantage that emptying and cleaning the tube are accomplished without difficulty, which is not the case if lead, rosin, etc., are used in a tube of this length. It is advisable to test the copper tubing before winding under sufficient hydraulic pressure to assure a reasonable factor of safety, and to test the completed regenerator at a lower pressure, but still somewhat higher than the normal working pressure.

\footnotetext{
A regenerator of this type has been described and illustrated by $\mathrm{H}$. Kammerlingh Onnes; Communicalians from the Phys. Lab. at the Univ. of Leiden, No. 87, 1903. Plate II, also No, rogb, 1909.

This method $w$ as devised by Dr. H. F. Stimson, of the Bureat of Standards. $60773^{\circ}-21-2$
} 


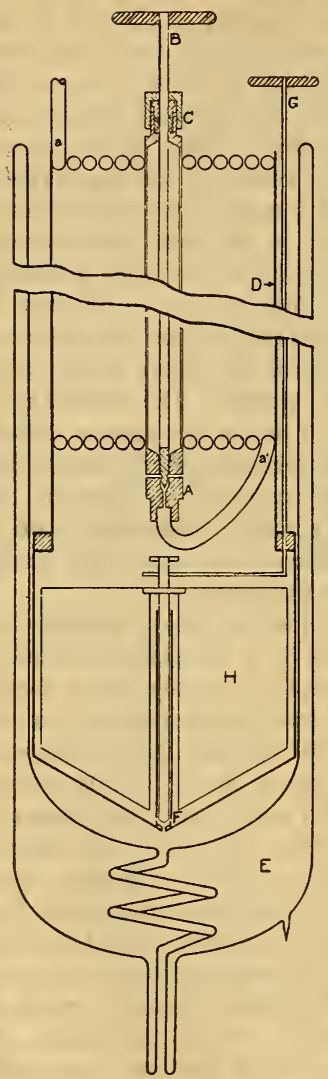

FYG. I.-Cross section, partly diagrammatic, of Hampson liquefier

$a a$, regenerator coil; $A$, expansion valve; $B$, valve stem; $C$, stuffing box; $D$, sheet metal casing: $E$, silvered glass Dewar cylinder; $F$, float valve; $G$, lifter for float valve; $H$, float

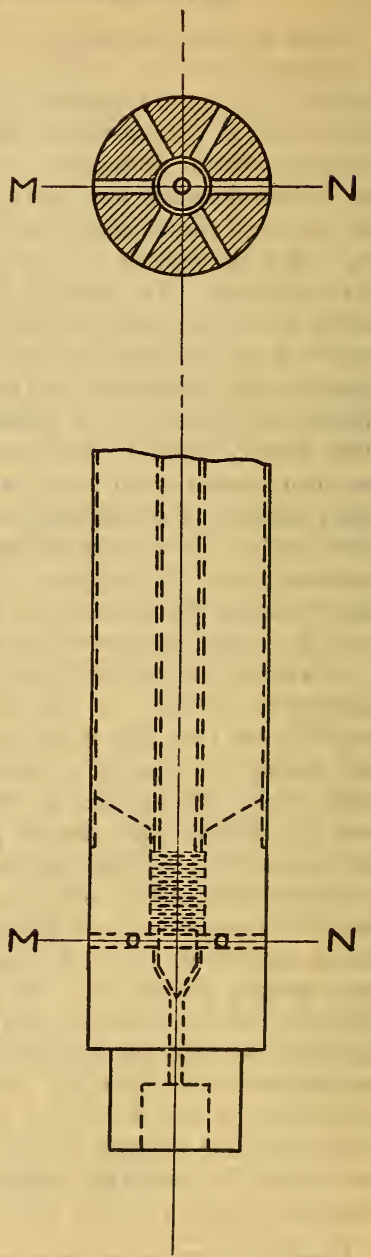

FIG. 3.-Expansion valve (twice actual size) 
Scientific Papers of the Bureau of Standards, Vol. 17

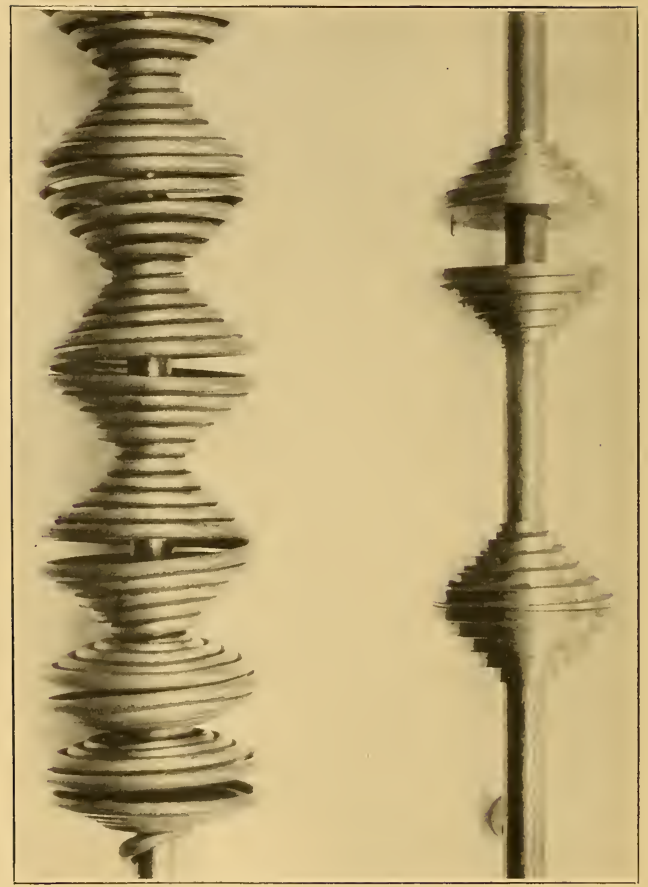

FIG. 2.-Regcnerator coil (at lcft) in course of construction and forms (at right) used in winding the coil. 
At the lower end of the core is soldered the expansion valve ( $A$, Fig. I), and at the lower end of this is silver soldered the end of the regenerator coil. Fig. 3 is a drawing of this expansion valve in detail, it being a needle valve of simple construction. A brass body and phosphor-bronze or gun-metal needle work well. The expansion valve is operated from above the top by means of the valve stem $(B)$, which is a German-silver tube extending up the core and through a stuffing box $(C)$, which holds the back pressure only. Any other type of valve that can be operated conveniently may be used, however, and even a fixed orifice, in the form of a few feet of copper capillary tube, has served in place of an expansion valve with fair success. It should be noted that the liquefier which was equipped with the capillary tube in place of an expansion valve did not permit of adjustment to accommodate variations in the speed of the compressor and also gave considerably more trouble by freezing and clogging, which will be discussed later.

A sheet-metal cylinder ( $D$, Fig. I) of thin German silver is made to fit tightly around the regenerator, open freely at the top to exhaust the gaseous air, and open through a small hole at the bottom to allow the liquid air to run out.

It is desirable to mount the complete liquefier in a suitable Dewar vessel $(E)$ having an outlet at the bottom. However, very satisfactory results have been obtained by using 3 to 4 inches of "fine regranulated cork" instead of a Dewar vessel. Fine regranulated cork is made commercially by grinding trimmings from cork board.

To work efficiently, the liquefier should discharge air in the liquid state from the bottom of the sheet-metal cylinder mentioned above, and all of the cold vapor should be forced up over the regenerator coil and out at the top. To accomplish this either a fixed, an adjustable, or an automatic outlet valve must be arranged at the bottom. A fixed opening naturally works with least efficiency, because some cold vapor escapes with the liquid and is a direct loss. An adjustable opening requires attention while running. An automatic float valve $(F)$ can be made to give high efficiency and very satisfactory service, but care must be taken in its design. It has been found that the violent turbulence of the air around the expansion valve and in the vicinity of the float $(H)$ may lift the float against gravity, hold the valve open, and prevent its operation. It is therefore necessary to install baffles to screen the float. No entirely satisfactory arrangement 
of baffles has been found, although several layers of fine copper gauze around the expansion valve serve fairly well. It is also necessary to remove moisture which will collect at the float valve when the liquefier is not in use and which would freeze if not removed when the apparatus was put into operation. If some mochanieal means $(G)$ is provided whereby the float valve can be lifted and held open while dry air is circulated through the liquefier just before starting a run, all moisture can thus be removed and the float mechanism will be free to open and close automatically. No ferrous metal should be incorporated in this part of the liquefier, as rusting would subsequently cause trouble.

When the liquid-air plant is in operation, the air after compression is cooled with running water to approximately $20^{\circ} \mathrm{C}$. Since the Joule-Thomson effect is greater at lower temperatures, it is very desirable to further cool the air before it reaches the liquefier, which is done by immersing a line of copper tubing in a tank containing ice and salt. Should a refrigerating plant producing a still lower temperature be available-using, for example, ammonia or carbon dioxide-a precooler should be included in the liquefier above the regenerator coil.

\section{PURIFICATION OF AIR}

It is also necessary that some means be provided for purifying the air by removing the water vapor and carbon dioxide. Most of the water vapor is condensed during compression, and a trap is located to catch this water, which can be drawn off at intervals, together with the oil used for lubrication in the compressor. The air is subsequently passed through a container, where it bubbles through a solution of sodium hydroxide, which removes carbon dioxide, and then passes over calcium chloride, which removes water vapor. This method is very effective, delivering air which is purer than is actually needed for normal conditions of liquefaction by the Hampson process. ${ }^{4}$ Calcium hydroxide in water (milk of lime) may be used to remove carbon dioxide, and calcium oxide (quicklime) may be used to remove water vapor, if preferred. If only one purifier is available, it can be used with dry sodium hydroxide in the lump or stick form, as this reacts with both carbon dioxide and water vapor.

\footnotetext{
1 The compressed-air plant at the Bureau of Standards is also used for refrigerating low-temperature apparatus, such as expansimeters and calorimeters, where a steady flow of air, without clogging, can be maintained for several hours at a time. This work demands that a higher degree of purity be attained than would be required for liquefaction alone.
} 
These purifiers are one of the chief sources of danger in a plant of this kind, as they contain comparatively large volumes of air under high pressure. They should, therefore, be designed with a considerable factor of safety and should be tested under hydraulic pressure considerably in excess of the working pressure. Each stage of the compressor is equipped with a spring safety valve, the one on the last stage also serving as the safety valve which protects the liquefier and purifiers as well as the compressor. It is suggested that a check valve, resembling the outlet valve of the last stage of the compressor, be placed in the air line between the first purifier and the high-pressure safety valve on the compressor to prevent a possible back flow of air in case of accident to this safety valve or to the compressor outlet valve and the gasket under the high-pressure cylinder head. If they should give way while pressure is on, any solution in the purifier would be atomized into the laboratory through the rupture unless such a check valve is provided.

A purifier for removing carbon dioxide may be placed in the intake line to the compressor, but as the volume of the air in the intake line is much greater than that in the high-pressure line more surface of the reagent must be exposed. When a purifier of proper size is placed in the intake line, it is effective for removing carbon dioxide, but very unsuitable for removing water vapor, because much water can be removed during compression, as mentioned above. Should any purifier be placed in the intake line, care must be taken to thoroughly filter the air before allowing it to enter the compressor, otherwise particles of the reagent used will be carried by the current of air into the valves of the compressor, eventually causing trouble. It is desirable, if feasible, to deliver to the intake of the compressor the exhaust air from the liquefier, because this air, having passed through the purifiers, has had most of the water and carbon dioxide removed.

When starting a Hampson liquid-air plant after pressure has been built up but before any liquid has been produced, there will be a very definite rate of flow of air through the apparatus. If the liquid-air outlet valve is closed before any liquid has been produced and the high-pressure gauge is watched closely, it will be observed that there is a definite time at which the pressure drops, indicating that the air flow has slightly increased. This corresponds with the first production of liquid. The mass rate of flow of partially liquefied air is probably greater than that of 
gaseous air under the same conditions, owing to the greater density of liquid air. This greater density, however, is accompanied by lower velocity of flow, which favors the deposition of ice or solid carbon dioxide at the point in the expansion valve or its vicinity at which liquefaction occurs, resulting in partial or complete clogging. To remove the obstruction, it is necessary to temporarily change the point where liquefaction is taking place to a point farther on in the air current, thereby increasing the velocity at the point where clogging occurred and sweeping the obstruction from its point of lodgment. This can be accomplished by momentarily opening the expansion valve a little and then reclosing it to its proper position. If the expansion valve is frozen in place and can not be moved, the same effect may be obtained by allowing some of the expanded air to escape directly out of the bottom of the apparatus (through the liquid-air outlet valve), thereby raising the temperature of the air in the expansion valve slightly. This causes a temporary vaporization and a consequent increase in velocity which will clear the valve of the accumulation of snow.

The difficulty just described does not ordinarily occur when the purifying train is in proper working order, because slight impurities will pass through the liquefier without causing obstruction and will be collected with the liquid air. Pure liquid air is clear and transparent, but when a very noticeable white precipitate is observed it may be taken as an indication that the reagents are exhausted and need to be replaced.

\section{DESCRIPTION OF BUREAU PLANT}

The following brief specifications give a general idea of the size and output of the Bureau of Standards liquid-air plant. The four-stage compressor, driven by a 35 -horsepower direct coupled steam engine, has a rated capacity of 75 cubic feet of free air per minute. The container for the sodium hydroxide solution used for removing the carbon dioxide is 8 feet long and $4 \frac{1}{2}$ inches in inside diameter and is placed in a vertical position, the air entering at the bottom and bubbling up through the solution. This container is nearly filled with pieces of broken marble, which cause better contact of the air with the solution and also prevent the carrying over of spray. About I gallon of a 25 per cent solution is used, which half fills the container. The flanged ends are bolted on, compressing a red fiber ring, which seals air-tight. Three 
calcium chloride purifiers, each 3 feet long and 2 inches in inside diameter, are used in series for removing water vapor. They are placed in a vertical position, and the air enters each successively from the bottom. A drain is located at the bottom of each tube for drawing off the calcium chloride solution which is formed during use. About 5 pounds of lump anhydrous calcium chloride are used to fill the three purifiers, and when replenishment is necessary only the first purifier in the series is removed and refilled. This refilled purifier is then replaced as the third one in the series, thus economizing on the reagent. All the purifiers are of seamless steel tubing. Connections to the purifiers are made by means of ball-and-socket unions of the type used on high-pressure gas cylinders.

The compressed air after being purified at ordinary temperature is cooled to about $0^{\circ} \mathrm{C}$ by a circulation of calcium chloride brine (cooled by an ammonia plant) and then to from -20 to $-35^{\circ} \mathrm{C}$ by a $\mathrm{CO}_{2}$ cycle.

The regenerator coil consists of about 200 feet of copper tubing of $\frac{3}{16}$ inch outside diameter and 0.035 -inch wall, mounted on a $1 / 2$-inch core. This coil when mounted is about 18 inches long and 3 inches in outside diameter and is completely jacketed by a glass Dewar vessel. An automatic float delivery valve is installed with mechanical adjustment for holding it open when desired. When working at full capacity, the plant produces 3 gallons of liquid air per hour. The general operation of the plant indicates that the above proportions are approximately correct.

Fig. 4 shows the construction of a very serviceable needle valve, which will be convenient in a laboratory where high-pressure air lines are desired for various purposes or where there is more than one liquefier available. This type of valve has been used at the Bureau of Standards for many years, but the origin of its design is not known. The body is usually of brass and the needle of steel, but where there is a possibility of the presence of moisture phosphor bronze is recommended in place of steel. The highpressure lines are connected to the valve by means of unions, one of which is represented. A thin gasket of pressboard is placed between the flat faces $(a)$ and $(b)$ and compressed air-tight by the nut $(c)$. The copper line is joined with silver solder at $(d)$.

Acknowledgments.-Many of the features described are similar to those of other commercial liquid air plants. The development of the liquid-air plant at the Bureau of Standards is due largely 
to F. S. Durston and the late T. B. Ford, who were formerly in charge. Acknowledgment is also due to Dr. C. W. Kanolt, at present in charge of the cryogenic laboratory, for a careful criticism of the manuscript.
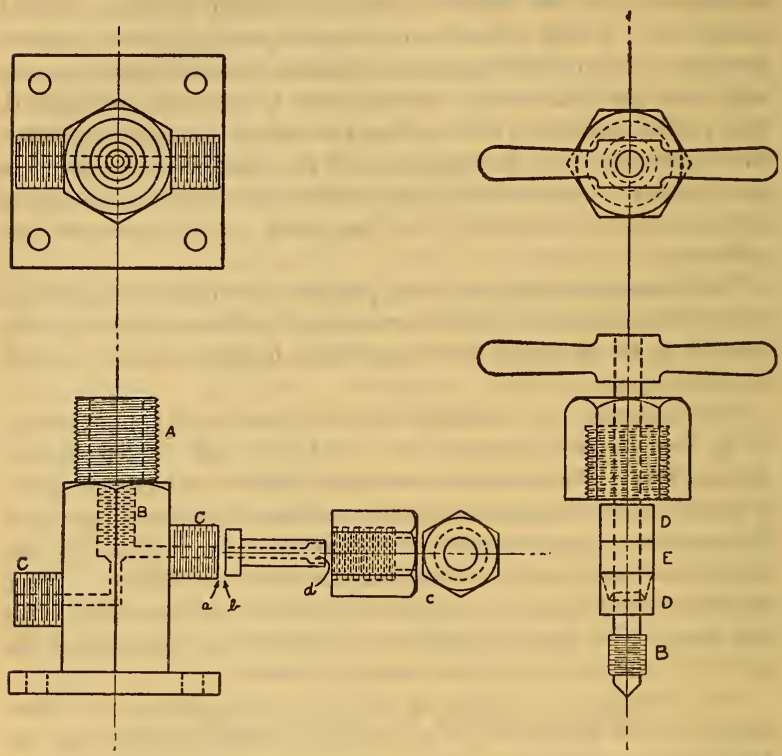

FIG. 4.-Needle valve for gases under high pressure

$A, x^{\prime \prime} \times 14$ thread; $B, 7 / 6^{\prime \prime} \times 20$ thread; $C, \mathrm{X}^{\prime \prime} \times 20$ thread; $D$, brass packing rings; $E$, leather packing; $b, c$, union connection

\section{SUMMARY}

The design and construction of laboratory apparatus for liquefying air by the Hampson process are described. Brief descriptions of necessary or useful accessories, such as purifiers for the air, precoolers, and a high-pressure needle valve, are included.

WASHINGTON, May I I, I920. 